\title{
Influência de porta-enxertos na resistência de mudas de cajueiro ao estresse salino
}

\author{
Sérgio Luiz Ferreira-Silva( ${ }^{(1)}$, Eduardo Luiz Voigt( ${ }^{(1)}$, Ricardo Almeida Viégas( ${ }^{(2)}$, João Rodrigues de Paiva ${ }^{(3)}$ \\ e Joaquim Albenísio Gomes Silveira ${ }^{(1)}$
}

\begin{abstract}
(1)Universidade Federal do Ceará, Departamento de Bioquímica e Biologia Molecular, Caixa Postal 6020, CEP 60451-970 Fortaleza, CE. E-mail: agrosergol@yahoo.com.br, elvoigt@yahoo.com.br, silveira@ufc.br (2)Universidade Federal de Campina Grande, Departamento de Engenharia Florestal, Caixa Postal 64, CEP 58700-970 Patos, PB. E-mail: raviegas@uol.com.br ${ }^{(3)}$ Embrapa Agroindústria Tropical, Caixa Postal 3761, CEP 60511-110 Fortaleza, CE. E-mail: paiva@cnpat.embrapa.br
\end{abstract}

Resumo - O objetivo deste trabalho foi avaliar a influência de porta-enxertos na resistência de mudas de cajueiro (Anacardium occidentale L.) à salinidade. As mudas foram obtidas pela enxertia do clone BRS 226 sobre os porta-enxertos CAPI 4, CCP 09 e BRS 226. Foram expostas a meio hidropônico sem $\mathrm{NaCl}$ (controle) ou com $\mathrm{NaCl} 200 \mathrm{mM}$ (tratamento salino), sob condições controladas de temperatura, umidade e luminosidade, durante 12 dias. O delineamento foi o inteiramente casualizado, em esquema fatorial 3x2 (três combinações de enxerto/ porta-enxerto e duas concentrações de $\mathrm{NaCl}$ ), com quatro repetições. Foram determinados a concentração de $\mathrm{Na}^{+}, \mathrm{Cl}, \mathrm{K}^{+}$e solutos orgânicos e os sintomas visuais de toxicidade nas folhas. Os conteúdos de $\mathrm{Na}^{+} \mathrm{ell}^{-}$a relação $\mathrm{K}^{+} / \mathrm{Na}^{+}$e as concentrações de aminoácidos e de prolina livres nas folhas tiveram relação direta com os sintomas visuais de toxicidade. Os porta-enxertos CAPI 4, CCP 09 e BRS 226 foram classificados como sensível, intermediário e resistente à salinidade elevada, respectivamente. Essa variação foi decorrente da influência do porta-enxerto na partição do $\mathrm{Na}^{+} \mathrm{e}$ do $\mathrm{Cl}^{-}$.

Termos para indexação: Anacardium occidentale, enxertia, estresse salino, solutos orgânicos.

\section{Influence of rootstocks on the resistance of cashew plantlets to salt stress}

\begin{abstract}
The aim of this work was to evaluate the influence of cashew (Anacardium occidentale L.) rootstocks on salt resistance in grafted plantlets. The plantlets were obtained by grafting the BRS 226 scion on CAPI 4, CCP 09 and BRS 226 rootstocks, and were exposed to nutrient solution without $\mathrm{NaCl}$ (control) or with $200 \mathrm{mM}$ $\mathrm{NaCl}$ (salt treatment) in hydroponics under controlled temperature, humidity, and luminosity conditions during 12 days. The experimental design was a completely randomized in factorial arrangement $3 \times 2$ (three scion/rootstock combinations, two $\mathrm{NaCl}$ concentrations) with four replicates. $\mathrm{Na}^{+}, \mathrm{Cl}^{-} \mathrm{K}^{+}$and organic solute concentrations and toxicity symptoms on leaves were determined. $\mathrm{Na}^{+}$and $\mathrm{Cl}^{-}$contents, the $\mathrm{K}^{+} / \mathrm{Na}^{+}$ratio and the free amino acid and proline concentrations in leaves were directly related to the visual toxicity symptoms. The rootstocks were classified as sensitive (CAPI 4), intermediate (CCP 09), and resistant (BRS 226) to high salinity. This variation was a consequence of the rootstock influence on $\mathrm{Na}^{+}$and $\mathrm{Cl}^{-}$partitioning.
\end{abstract}

Index terms: Anacardium occidentale, grafting, salt stress, organic solutes.

\section{Introdução}

Devido à expansão das áreas salinizadas em todo o mundo, o estresse salino tem se tornado um fator limitante da produtividade agrícola (Munns \& Tester, 2008). Em regiões áridas e semiáridas, esse problema pode ser agravado por outros estresses ambientais, incluindo baixa disponibilidade de água, temperaturas elevadas e elevada evapotranspiração (Viégas et al., 2001). O estresse salino afeta a produtividade em razão de alterações nas relações hídricas (Bray et al., 2000) e no metabolismo das plantas (Maathuis \& Amtmann, 1999; Apse \& Blumwald, 2007).

Os efeitos do sal sobre as plantas são evidenciados por sintomas de toxicidade, como clorose ou necrose foliar, acompanhados pela acumulação excessiva de $\mathrm{Na}^{+}$ou de $\mathrm{Cl}^{-}$nos tecidos (Wahome et al., 2001; GarcíaSánchez et al., 2002). O aumento da concentração de $\mathrm{Na}^{+}$nos tecidos foliares pode afetar processos fisiológicos e bioquímicos dependentes de $\mathrm{K}^{+}$, como a abertura estomática, a fotossíntese, a respiração e a síntese de proteínas, em virtude da similaridade físico- 
química entre esses íons (Maathuis \& Amtmann, 1999; Apse \& Blumwald, 2007). A concentração elevada de $\mathrm{Cl}^{-}$no meio de crescimento, por sua vez, pode interferir na absorção de $\mathrm{NO}_{3}{ }^{-}$e na osmorregulação (White \& Broadley, 2001). Assim, a toxicidade iônica pode ser evidenciada por alterações nas razões $\mathrm{K}^{+} / \mathrm{Na}^{+}, \mathrm{Ca}^{2+} / \mathrm{Na}^{+}$ e $\mathrm{NO}_{3}{ }^{-} / \mathrm{Cl}^{-}$dos tecidos, o que compromete a homeostase iônica nas células (Grattan \& Grieve, 1999; Apse \& Blumwald, 2007).

Com o intuito de amenizar os danos causados pelo estresse salino sobre o rendimento agrícola, diversas estratégias têm sido utilizadas. Essas estratégias incluem a correção dos solos, considerada dispendiosa e temporária (Dasgan et al., 2002), e o melhoramento genético para a obtenção de genótipos mais resistentes, considerado laborioso e complexo em função do caráter multifatorial da resistência (Flowers, 2004). A utilização dos mecanismos de resistência ao estresse salino pode constituir a abordagem mais viável e imediata para reduzir os impactos da salinidade sobre as culturas. Entre esses mecanismos, a exclusão foliar de $\mathrm{Na}^{+}$e $\mathrm{Cl}^{-}$é fundamental para a caracterização da resistência à salinidade (Ashraf \& Ahmad, 2000).

Os mecanismos associados à exclusão foliar de $\mathrm{Na}^{+}$ e $\mathrm{Cl}^{-}$incluem tanto a seletividade da absorção radicular quanto a restrição da transferência para a parte aérea (Maathuis \& Amtmann, 1999). Esses mecanismos minimizam a toxicidade iônica nos tecidos foliares e são essenciais para a resistência ao estresse salino em roseira (Wahome et al., 2001), porta-enxertos de citros (García-Sánchez et al., 2002), tomateiro (Estañ et al., 2005) e videira (Paranychianakis \& Angelakis, 2008). A utilização de porta-enxertos excluidores de $\mathrm{Na}^{+}$e $\mathrm{Cl}^{-}$pode resultar em plantas enxertadas mais resistentes à salinidade, desde que o genótipo apresente variabilidade genética quanto ao caráter excluidor desses íons (Ashraf \& Ahmad, 2000).

$\mathrm{O}$ cajueiro (Anacardium occidentale L.) é uma espécie amplamente cultivada no Semiárido Nordestino e representa uma importante fonte de emprego e renda nos estados do Ceará, Piauí e Rio Grande do Norte, os quais são localizados em áreas sujeitas à salinização dos solos e da água. Apesar de o cajueiro ser cultivado em condições semiáridas e apresentar resistência moderada à salinidade, diversos estudos demonstram que o estresse salino afeta severamente as fases de germinação (Voigt et al., 2009), crescimento inicial (Ferreira-Silva et al., 2008), enxertia (Bezerra et al., 2002) e prefloração (Carneiro et al., 2007), além de induzir distúrbios metabólicos relacionados à mobilização de reservas (Voigt et al., 2009), à fotossíntese (Bezerra et al., 2007), ao metabolismo do nitrogênio (Viégas et al., 2004) e à homeostase iônica (Viégas et al., 2001).

O objetivo deste trabalho foi avaliar a influência de porta-enxertos na resistência de mudas de cajueiro à salinidade.

\section{Material e Métodos}

As mudas foram obtidas e cultivadas em condições de casa de vegetação no Departamento de Bioquímica e Biologia Molecular da Universidade Federal do Ceará, Fortaleza, CE ( $3^{\circ} 45^{\prime} 47^{\prime \prime} \mathrm{S}, 38^{\circ} 31^{\prime} 23^{\prime \prime} \mathrm{W}$, ao nível do mar), de junho a novembro de 2007. As plantas permaneceram expostas às condições naturais, com temperaturas entre $29 \pm 4^{\circ} \mathrm{C}$ ao dia e $25 \pm 2^{\circ} \mathrm{C}$ à noite, umidade relativa de $60 \pm 10 \%$, radiação fotossinteticamente ativa (PAR) de $600 \pm 200 \mu \mathrm{mol} \mathrm{m} \mathrm{m}^{-2} \mathrm{~s}^{-1} \mathrm{e}$ fotoperíodo médio de 12 horas.

Para a aplicação do tratamento salino, as mudas foram acondicionadas em câmara de crescimento com condições controladas, à temperatura de $28^{\circ} \mathrm{C}$, umidade relativa de $80 \%$, radiação fotossinteticamente ativa de $240 \mu \mathrm{mol} \mathrm{m} \mathrm{m}^{-2} \mathrm{e}$ fotoperíodo de 12 horas.

$\mathrm{O}$ delineamento foi o inteiramente casualizado, com tratamentos dispostos em fatorial $3 \times 2$ : três combinações de enxerto e porta-enxerto (BRS 226/CCP 09, BRS 226/ CAPI 4, BRS 226/BRS 226) e duas concentrações de $\mathrm{NaCl}(0$ e $200 \mathrm{mM})$. Foram realizadas quatro repetições, e cada unidade experimental consistiu de um vaso com uma muda enxertada.

As castanhas e os enxertos de cajueiro-anão precoce (Anacardium occidentale L.) foram fornecidos pela Embrapa Agroindústria Tropical, Fortaleza, CE. Os porta-enxertos foram obtidos a partir de castanhas dos clones CCP 09, CAPI 4 e BRS 226. O clone CCP 09 é um dos mais empregados em plantios comerciais (Barros et al., 2000), o CAPI 4 é cultivado em sequeiro no Estado do Piauí e o BRS 226 é recomendado para cultivo de sequeiro no Semiárido Nordestino (Paiva et al., 2002).

As castanhas foram desinfetadas em hipoclorito de sódio comercial $5 \%(\mathrm{v} / \mathrm{v})$ por $10 \mathrm{~min}$, lavadas com água destilada e germinadas em vermiculita, em vasos de 0,8 L. Durante a germinação, a umidade do substrato 
foi mantida próxima da capacidade de campo, por irrigações com água destilada. Em seguida, as plântulas foram irrigadas com solução nutritiva de Hoagland \& Arnon (1950) diluída 1:1 por 20 dias. Ao atingir o estádio fisiológico de oito folhas expandidas, as plântulas foram enxertadas por garfagem lateral no clone BRS 226.

Aproximadamente 110 dias pós-enxertia, as mudas foram transferidas para câmara de crescimento com condições controladas, para isolar o efeito da salinidade. Após 24 horas de aclimatação, foram transplantadas para solução nutritiva diluída 1:10, em vasos de 0,8 L. Após 24 horas de aclimatação ao meio líquido, as plantas foram expostas aos tratamentos controle (solução nutritiva sem $\mathrm{NaCl}$ ) e salino (solução nutritiva com $\mathrm{NaCl} 200 \mathrm{mM}$ ) por 12 dias. O tratamento salino foi aplicado de forma fracionada, com uma dose de $\mathrm{NaCl} 100 \mathrm{mM}$ nas primeiras 24 horas, seguida da dose final de $\mathrm{NaCl} 200 \mathrm{mM}$.

Durante a coleta, as mudas foram separadas em raiz, caule inferior (abaixo da enxertia), caule superior (acima da enxertia) e folhas. As folhas foram congeladas em $\mathrm{N}_{2}$ líquido, armazenadas a $-20^{\circ} \mathrm{C}$ e liofilizadas, para posterior determinação de solutos orgânicos e inorgânicos. Raízes e caules foram secos em estufa a $70^{\circ} \mathrm{C}$ por 72 horas, triturados em moinho, e a farinha foi utilizada nas determinações dos íons $\mathrm{Na}^{+}, \mathrm{K}^{+}$e $\mathrm{Cl}^{-}$.

Os teores de $\mathrm{Na}^{+}$e $\mathrm{K}^{+}$foram determinados por fotometria de chama, e de $\mathrm{Cl}^{-}$, por titulação com nitrato de prata $\left(\mathrm{AgNO}_{3}\right)$. O conteúdo de proteínas solúveis foi mensurado conforme Bradford (1976), com uso de albumina sérica bovina (BSA) como padrão. A concentração de aminoácidos livres totais (AALT) foi determinada de acordo com Peoples et al. (1989) pela curva padrão de glicina, e a concentração de prolina livre foi mensurada conforme Bates (1973) pela curva padrão de prolina. A clorofila foi determinada conforme Arnon (1949) e estimada pela expressão $\left(20,2 \mathrm{~A}_{645}+\right.$ $\left.8,02 \mathrm{~A}_{663}\right) \mathrm{V} / 1000 \mathrm{~W}$, em que A é a absorbância, $\mathrm{V}$ é o volume final do extrato $(5 \mathrm{~mL})$ e $\mathrm{W}$ é a massa de matéria seca em gramas.

Os dados foram submetidos à análise de variância, e as médias foram comparadas pelo teste de Tukey.

\section{Resultados e Discussão}

As mudas enxertadas de cajueiro-anão precoce exibiram sintomas visuais de toxicidade foliar a partir do sexto dia de tratamento salino, os quais foram agravados com o tempo de exposição. Os sintomas observados no clone BRS 226 enxertado sobre o CAPI 4 foram mais intensos se comparados com os verificados nas mudas enxertadas sobre CCP 09 e BRS 226 (autoenxertia). Nas mudas enxertadas em CAPI 4, ocorreu necrose na maior parte da área foliar, seguida de rápida senescência e abscisão aos 12 dias de tratamento. As mudas enxertadas em CCP 09, por sua vez, apresentaram clorose e pontos necróticos nas lâminas foliares, enquanto as mudas sobre BRS 226 exibiram apenas algumas áreas necróticas. Esses sintomas são geralmente associados à acumulação excessiva de $\mathrm{Na}^{+}$e $\mathrm{Cl}^{-}$nos tecidos foliares e à sensibilidade ao estresse salino em genótipos de roseira (Wahome et al., 2001), tomateiro (Dasgan et al., 2002) e videira (Paranychianakis \& Angelakis, 2008).

Os conteúdos de $\mathrm{Na}^{+}, \mathrm{Cl}^{-}$e $\mathrm{K}^{+}$foliar das mudas foram afetados pela concentração de $\mathrm{NaCl}$ e pelo porta-enxerto (Tabelas 1 e 2). Na ausência do $\mathrm{NaCl}$, os conteúdos de $\mathrm{Na}^{+}$e $\mathrm{Cl}^{-}$foliar (Tabela 2) não diferiram em função do porta-enxerto. No entanto, o tratamento salino aumentou o conteúdo de $\mathrm{Na}^{+}$foliar em 114, 71 e $66 \%$ nas mudas enxertadas em CAPI 4, CCP 09 e BRS 226, respectivamente, em relação aos respectivos controles. Além disso, o conteúdo de $\mathrm{Cl}^{-}$foliar foi incrementado em 231,270 e $224 \%$ nas mudas enxertadas em CAPI 4, CCP 09 e BRS 226, respectivamente. $\mathrm{Na}$ presença do sal, as mudas enxertadas em CAPI 4 e CCP 09 apresentaram conteúdo de $\mathrm{Na}^{+}$foliar 44 e $27 \%$ maior, respectivamente, em relação às mudas autoenxertadas. O conteúdo de $\mathrm{Cl}^{-}$foliar, por sua vez, foi 40 e $21 \%$ maior nas mudas enxertadas em CAPI 4 e CCP 09, respectivamente, em comparação com as

Tabela 1. Valores de F para os conteúdos de solutos orgânicos e inorgânicos em folhas de mudas de cajueiro-anão precoce do clone BRS 226 enxertado sobre diferentes porta-enxertos, cultivadas na ausência e presença de $\mathrm{NaCl} 200 \mathrm{mM}$, durante 12 dias.

\begin{tabular}{lccc}
\hline Soluto & Porta-enxerto & $\mathrm{NaCl}$ & Interação \\
\hline $\mathrm{Na}^{+}$ & $12,62^{* *}$ & $134,94^{* *}$ & $5,58^{* *}$ \\
$\mathrm{Cl}^{-}$ & $21,10^{* *}$ & $680,35^{* *}$ & $6,74^{* *}$ \\
$\mathrm{~K}^{+}$ & $34,85^{* *}$ & $4,78^{* *}$ & $1,19^{\text {ns }}$ \\
$\mathrm{PS}^{(1)}$ & $3,15^{\mathrm{ns}}$ & $0,28^{\mathrm{ns}}$ & $3,02^{\mathrm{ns}}$ \\
Clorofilas $^{\text {AALT }}$ & $7,80^{* *}$ & $1,50^{\text {ns }}$ & $154,44^{* *}$ \\
Prolina & $2,28^{\mathrm{ns}}$ & $21,78^{* *}$ & $4,59^{*}$ \\
\hline
\end{tabular}

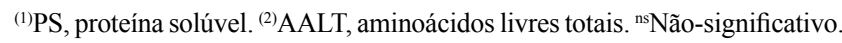
$* \mathrm{e} * *$ Significativo a 5 e $1 \%$ de probabilidade, respectivamente, pelo teste $\mathrm{F}$. 
autoenxertadas. $\mathrm{O}$ conteúdo de $\mathrm{K}^{+}$foliar (Tabela 2) não foi afetado pelo $\mathrm{NaCl}$ nas mudas enxertadas em CAPI 4 e CCP 09 , mas foi reduzido em 14\% nas mudas autoenxertadas.

A acumulação de $\mathrm{Na}^{+}$e $\mathrm{Cl}^{-}$em folhas de mudas enxertadas de cajueiro sob condições salinas foi fortemente influenciada pelo porta-enxerto. O portaenxerto BRS 226 apresentou maior capacidade de exclusão foliar de $\mathrm{Na}^{+}$e $\mathrm{Cl}^{-}$em comparação com os porta-enxertos CAPI 4 e CCP 09. Em outras espécies cultivadas, o padrão de acumulação de íons salinos nas folhas tem sido utilizado para a identificação de porta-enxertos excluidores. Em plantas de roseira expostas ao $\mathrm{NaCl}$, o porta-enxerto Rosa rubiginosa apresentou sintomas de toxicidade mais brandos, maior crescimento e menor acumulação foliar de $\mathrm{Na}^{+} \mathrm{e} \mathrm{Cl}^{-} \mathrm{em}$ comparação com o porta-enxerto $R$. chinensis Major, o que evidenciou que a maior capacidade excluidora de $R$. rubiginosa está relacionada com a resistência à salinidade (Wahome et al., 2001). De forma similar, plantas de citros enxertadas em porta-enxerto excluidor tangerineira Cleópatra (Citrus reshni Hort. ex Tanaka) apresentaram maior crescimento, maior eficiência fotossintética e de uso da água, associados ao menor conteúdo de $\mathrm{Na}^{+}$e $\mathrm{Cl}^{-}$foliar sob salinidade, em comparação com as plantas enxertadas em portaenxerto incluidor citranjeiro Carrizo [C. sinensis Osbeck x Poncirus trifoliata (L.) Raf.] (García-Sánchez et al., 2002).

Alguns trabalhos relatam a identificação de portaenxertos contrastantes quanto à exclusão foliar de $\mathrm{Na}^{+}$e $\mathrm{Cl}^{-}$em roseira (Wahome et al., 2001), citros (García-Sánchez et al., 2002), tomateiro (Estañ et al., 2005) e videira (Paranychianakis \& Angelakis, 2008).

Tabela 2. Conteúdo de $\mathrm{Na}^{+}, \mathrm{Cl}^{-}$e $\mathrm{K}^{+}\left(\mathrm{mmol} \mathrm{kg}{ }^{-1}\right)$ e relação $\mathrm{K}^{+} / \mathrm{Na}^{+}$em folhas de mudas de cajueiro-anão precoce do clone BRS 226 enxertado em diferentes porta-enxertos, cultivadas na ausência e presença de $\mathrm{NaCl} 200 \mathrm{mM}$, durante $12 \operatorname{dias}^{(1)}$.

\begin{tabular}{lccccc}
\hline Porta-enxerto & $\mathrm{NaCl}(\mathrm{mM})$ & $\mathrm{Na}^{+}$ & $\mathrm{Cl}^{-}$ & $\mathrm{K}^{+}$ & $\mathrm{K}^{+} / \mathrm{Na}^{+}$ \\
\hline CCP 09 & 0 & $307,22 \mathrm{c}$ & $159,62 \mathrm{~d}$ & $426,25 \mathrm{~b}$ & 1,38 \\
& 200 & $527,50 \mathrm{a}$ & $591,55 \mathrm{~b}$ & $422,84 \mathrm{~b}$ & 0,80 \\
CAPI 4 & 0 & $278,24 \mathrm{c}$ & $206,57 \mathrm{~d}$ & $341,00 \mathrm{c}$ & 1,22 \\
& 200 & $597,67 \mathrm{a}$ & $685,45 \mathrm{a}$ & $317,13 \mathrm{c}$ & 0,53 \\
BRS 226 & 0 & $249,26 \mathrm{c}$ & $150,35 \mathrm{~d}$ & $504,68 \mathrm{a}$ & 2,02 \\
& 200 & $414,17 \mathrm{~b}$ & $488,26 \mathrm{c}$ & $433,07 \mathrm{~b}$ & 1,04 \\
\hline
\end{tabular}

(1)Médias seguidas de letras iguais, na coluna, não diferem entre si a 5\% de probabilidade, pelo teste de Tukey.
Esses trabalhos demonstram a existência de variabilidade genética quanto ao caráter excluidor em diferentes espécies cultivadas, o que reforça a validade da identificação e da utilização desses genótipos na obtenção de plantas enxertadas mais resistentes ao sal. No presente estudo, embora apenas três porta-enxertos de cajueiro-anão precoce tenham sido avaliados, foi demonstrada a existência de contrastes em relação à exclusão foliar de $\mathrm{Na}^{+} \mathrm{e} \mathrm{Cl}^{-}$.

Além da acumulação excessiva de $\mathrm{Na}^{+} \mathrm{e} \mathrm{Cl}^{-}$associada a sintomas visuais de toxicidade foliar, o tratamento salino também induziu alterações na concentração de alguns compostos orgânicos indicadores de homeostase metabólica (Tabelas 1 e 3). Embora os conteúdos de proteínas solúveis e clorofilas totais (Tabela 3) não tenham apresentado modificações em resposta ao $\mathrm{NaCl}$, o conteúdo de clorofilas foi $60 \%$ maior nas mudas autoenxertadas em relação às demais combinações, independentemente do $\mathrm{NaCl}$. O conteúdo foliar de aminoácidos livres totais (AALT) nas mudas enxertadas em CCP 09 e BRS 226 não foi alterado pelo tratamento salino, mas aumentou $80 \%$ nas mudas enxertadas em CAPI 4 em relação aos respectivos controles. O conteúdo de prolina foliar nas mudas salinizadas enxertadas em CAPI 4, CCP 09 e BRS 226 foi aumentado $11,4,3,8$ e 2,2 vezes, respectivamente, em comparação com os respectivos controles. Entre as mudas salinizadas, aquelas enxertadas em CCP 09 e CAPI 4 apresentaram conteúdo foliar de prolina 36 e $112 \%$ maior, respectivamente, em relação às mudas autoenxertadas.

O aumento dos solutos orgânicos em tecidos de plantas expostas à seca ou ao déficit hídrico induzido pelo sal tem sido atribuído ao ajustamento osmótico

Tabela 3. Conteúdo de proteínas solúveis (PS) e clorofilas totais $\left(\mathrm{mg} \mathrm{g}^{-1}\right)$ e de aminoácidos livres totais (AALT) e prolina livre ( $\mathrm{mmol} \mathrm{kg}^{-1}$ ) em folhas de mudas de cajueiroanão precoce do clone BRS 226, enxertado em diferentes porta-enxertos, cultivadas na ausência e presença de $\mathrm{NaCl}$ $200 \mathrm{mM}$, durante 12 dias $^{(1)}$.

\begin{tabular}{|c|c|c|c|c|c|c|}
\hline \multirow[t]{2}{*}{ Solutos } & \multicolumn{2}{|c|}{ CCP 09} & \multicolumn{2}{|c|}{ CAPI 4} & \multicolumn{2}{|c|}{ BRS 226} \\
\hline & Controle & $\mathrm{NaCl}$ & Controle & $\mathrm{NaCl}$ & Controle & $\mathrm{NaCl}$ \\
\hline$\overline{\mathrm{PS}}$ & $73,49 a$ & $70,20 \mathrm{a}$ & $97,52 \mathrm{a}$ & $81,41 \mathrm{a}$ & $61,08 \mathrm{a}$ & $88,91 \mathrm{a}$ \\
\hline Clorofilas & $1,84 \mathrm{~b}$ & $1,66 \mathrm{~b}$ & $1,56 \mathrm{~b}$ & $1,37 \mathrm{~b}$ & $2,60 \mathrm{a}$ & $2,36 \mathrm{a}$ \\
\hline AALT & $57,04 b$ & $74,02 b$ & $56,12 b$ & $102,84 \mathrm{a}$ & $67,57 \mathrm{~b}$ & $77,57 b$ \\
\hline Prolina & $1,20 \mathrm{c}$ & $4,63 \mathrm{~b}$ & $0,63 \mathrm{~d}$ & $7,17 \mathrm{a}$ & $1,51 \mathrm{c}$ & $3,38 b$ \\
\hline
\end{tabular}

${ }^{(1)}$ Médias seguidas de letras iguais, na linha, não diferem entre si a 5\% de probabilidade, pelo teste de Tukey. 
e à proteção de estruturas celulares (Bray et al., 2000). Diversos estudos têm demonstrado que a acumulação de solutos compatíveis, como prolina e glicina-betaína, está relacionada à resistência a esses estresses abióticos, o que indica que esses solutos têm papel na osmoproteção (Ashraf \& Harris, 2004). No entanto, outros estudos têm indicado que a acumulação de solutos orgânicos em condições ambientais adversas deve estar mais relacionada a distúrbios metabólicos do que ao ajustamento osmótico (Lutts et al., 1996).

$\mathrm{O}$ aumento do conteúdo foliar de prolina em plantas jovens de cajueiro expostas ao $\mathrm{NaCl}$ ocorreu associado a sintomas de toxicidade iônica, restrição do crescimento da parte aérea, acumulação de AALT, redução do conteúdo de proteínas solúveis e aumento da atividade proteolítica (Silveira et al., 2003; Viégas et al., 2004). Assim, o aumento do conteúdo foliar de prolina em resposta ao $\mathrm{NaCl}$ não pode ser atribuído ao ajustamento osmótico nessa espécie.

Os sintomas de toxicidade mais intensos, observados nas mudas enxertadas nos porta-enxertos CAPI $4 \mathrm{e}$ CCP 09, ocorreram associados ao maior conteúdo foliar de $\mathrm{Na}^{+}, \mathrm{Cl}^{-}$, AALT e prolina livre, em comparação com as mudas autoenxertadas. Esses resultados evidenciam a ocorrência de distúrbios metabólicos induzidos pelo $\mathrm{NaCl}$, o que indica maior sensibilidade dos porta-enxertos CAPI 4 e CCP 09 ao sal. Além desses indicadores, a relação $\mathrm{K}^{+} / \mathrm{Na}^{+}$foliar nas mudas enxertadas com esses porta-enxertos alcançou valores inferiores a 1,0 em estresse salino (Tabela 2), o que indica desequilíbrio nutricional (Maathuis \& Amtmann, 1999) e reforça a maior intensidade de toxicidade iônica nas combinações BRS 226/CAPI 4 e BRS 226/CCP 09 em relação às mudas enxertadas em BRS 226.
As mudas apresentaram padrão de partição de $\mathrm{Na}^{+}$e $\mathrm{Cl}^{-}$dependente dos porta-enxertos utilizados. $\mathrm{O}$ conteúdo de $\mathrm{Na}^{+}$e $\mathrm{Cl}^{-}$aumentou cerca de duas e quatro vezes, respectivamente, no caule superior (acima da enxertia), no caule inferior (abaixo da enxertia) e na raiz das mudas enxertadas em CCP 09 e BRS 226 no tratamento salino em comparação aos respectivos controles (Tabela 4). Nas mudas enxertadas em CAPI 4, no entanto, o tratamento salino causou um incremento de três vezes no conteúdo de $\mathrm{Na}^{+}$do caule inferior e da raiz e ocasionou um aumento de cinco vezes no conteúdo de $\mathrm{Cl}^{-}$do caule superior e inferior. Além disso, as mudas autoenxertadas apresentaram menor conteúdo de $\mathrm{Na}^{+}$no caule superior e inferior, bem como menor conteúdo de $\mathrm{Cl}^{-}$no caule inferior e na raiz, se comparadas às mudas enxertadas em CAPI 4 e CCP 09. $\mathrm{O}$ conteúdo de $\mathrm{K}^{+}$no caule e nas raízes não foi afetado pelo $\mathrm{NaCl}$ e apresentou valores $30 \%$ menores nas mudas enxertadas sobre o porta-enxerto CAPI 4 , em relação às outras combinações. No caule superior, o conteúdo de $\mathrm{K}^{+}$foi cerca de duas vezes maior se comparado ao das raízes e do caule inferior, independentemente do portaenxerto e do $\mathrm{NaCl}$.

$\mathrm{O}$ padrão de partição de $\mathrm{Na}^{+} \mathrm{e} \mathrm{Cl}^{-}$entre as diferentes partes da planta tem sido relacionado com a resistência à salinidade (Ashraf \& Ahmad, 2000). Espécies e genótipos de glicófitas são considerados mais resistentes quando apresentam menores conteúdos de íons salinos nas folhas em relação às partes basais, o que evita a toxicidade iônica nos tecidos fotossintéticos (Munns \& Tester, 2008). Esse padrão de partição pode estar relacionado aos mecanismos seletivos de absorção e acumulação de íons salinos no sistema radicular, assim como a restrição da sua transferência para a parte aérea (Maathuis \& Amtmann, 1999). Por exemplo, porta-

Tabela 4. Conteúdos de $\mathrm{Na}^{+}, \mathrm{Cl}^{-}$e $\mathrm{K}^{+}\left(\mathrm{mmol} \mathrm{kg}{ }^{-1}\right)$ em raízes e caules de mudas de diferentes combinações de enxerto (clone BRS 226) e porta-enxertos (clones CCP 09, CAPI 4 e BRS 226) de cajueiro-anão precoce, cultivadas na ausência e presença de $\mathrm{NaCl} 200 \mathrm{mM}^{(1)}$.

\begin{tabular}{|c|c|c|c|c|c|c|c|}
\hline \multirow[t]{2}{*}{ Íon } & \multirow[t]{2}{*}{ Parte da planta } & \multicolumn{2}{|c|}{ CCP 09} & \multicolumn{2}{|c|}{ CAPI 4} & \multicolumn{2}{|c|}{ BRS 226} \\
\hline & & Controle & $\mathrm{NaCl}$ & Controle & $\mathrm{NaCl}$ & Controle & $\mathrm{NaCl}$ \\
\hline \multirow[t]{3}{*}{$\overline{\mathrm{Na}^{+}}$} & Caule superior & $231,87 \mathrm{c}$ & $544,89 a$ & $237,89 \mathrm{c}$ & $556,48 \mathrm{a}$ & $202,88 \mathrm{c}$ & $434,75 b$ \\
\hline & Caule inferior & $208,23 d$ & $469,53 b$ & $168,26 \mathrm{~d}$ & $533,29 a$ & $168,38 d$ & $394,17 \mathrm{c}$ \\
\hline & Raiz & $249,26 \mathrm{~b}$ & $527,51 \mathrm{a}$ & $162,31 \mathrm{~b}$ & $486,92 \mathrm{a}$ & $226,07 b$ & $486,92 \mathrm{a}$ \\
\hline \multirow[t]{3}{*}{$\overline{\mathrm{Cl}^{-}}$} & Caule superior & $140,80 \mathrm{c}$ & $629,11 a$ & $120,11 \mathrm{c}$ & $594,37 \mathrm{ab}$ & $122,06 \mathrm{c}$ & $572,77 b$ \\
\hline & Caule inferior & $103,28 \mathrm{c}$ & $431,92 a$ & $89,33 c$ & $507,04 \mathrm{a}$ & $84,50 \mathrm{c}$ & $366,20 b$ \\
\hline & Raiz & $112,67 \mathrm{c}$ & $460,09 a$ & $92,33 \mathrm{c}$ & $403,76 \mathrm{ab}$ & $91,94 \mathrm{c}$ & $356,81 \mathrm{~b}$ \\
\hline \multirow[t]{3}{*}{$\overline{\mathrm{K}^{+}}$} & Caule superior & $351,23 a$ & $351,23 a$ & $248,93 b$ & $248,93 b$ & $392,15 \mathrm{a}$ & $388,74 a$ \\
\hline & Caule inferior & $146,63 \mathrm{a}$ & $132,99 a$ & $132,99 \mathrm{a}$ & $122,76 a$ & $163,68 \mathrm{a}$ & $170,50 \mathrm{a}$ \\
\hline & Raiz & $184,14 \mathrm{a}$ & $190,96 \mathrm{a}$ & $150,04 \mathrm{a}$ & $153,45 \mathrm{a}$ & $160,27 \mathrm{a}$ & $160,27 \mathrm{a}$ \\
\hline
\end{tabular}

${ }^{(1)}$ Médias seguidas de letras iguais, na linha, não diferem entre si a 5\% de probabilidade, pelo teste de Tukey. 
enxertos de citros com maior capacidade de retenção de $\mathrm{Na}^{+}$e $\mathrm{Cl}^{-}$no sistema radicular conferem maior resistência à toxicidade foliar, associada ao menor conteúdo desses íons nas folhas (García-Sánchez et al., 2002). No entanto, a menor acumulação de $\mathrm{Na}^{+}$ e $\mathrm{Cl}^{-}$nas folhas das mudas autoenxertadas, verificada no presente trabalho, não pode ser atribuída à retenção desses íons no sistema radicular. Desse modo, o caráter excluidor do porta-enxerto BRS 226 parece estar mais associado a mecanismos seletivos de absorção que à acumulação preferencial de $\mathrm{Na}^{+} \mathrm{e} \mathrm{Cl}^{-}$na raiz.

O porta-enxerto BRS 226 apresentou maior habilidade para excluir $\mathrm{Na}^{+} \mathrm{e} \mathrm{Cl}^{-}$das folhas e das partes basais em comparação com os porta-enxertos CAPI 4 e CCP 09, o que resulta em uma maior capacidade para aclimatação ao estresse salino. Também Paiva et al. (2008) encontrou melhor desempenho do clone BRS 226 do que do clone CAC 35 em condições semiáridas. Dessa forma, o clone BRS 226 tem se mostrado uma boa opção como enxerto ou portaenxerto para cultivo em regiões expostas a condições adversas de restrição hídrica e salinidade.

\section{Conclusões}

1. A acumulação de $\mathrm{Na}^{+}$e $\mathrm{Cl}^{-}$e a relação $\mathrm{K}^{+} / \mathrm{Na}^{+}$, em folhas de mudas enxertadas de cajueiro cultivadas em meio salinizado, são dependentes do porta-enxerto utilizado quando a copa é do clone BRS 226.

2. A maior resistência à salinidade das mudas autoenxertadas (BRS 226/BRS 226) e a maior sensibilidade das mudas do clone BRS 226 enxertadas em CAPI 4 estão relacionadas à capacidade de exclusão foliar de $\mathrm{Na}^{+}$e $\mathrm{Cl}^{-}$.

3. A sensibilidade de mudas de cajueiro ao estresse salino está associada aos conteúdos foliares de aminoácidos livres totais e prolina.

\section{Agradecimentos}

Ao Conselho Nacional de Desenvolvimento Cientifico e Tecnológico, pela concessão de bolsas; à Fundação Cearense de Amparo à Pesquisa, pelo apoio financeiro.

\section{Referências}

APSE, M.P.; BLUMWALD, E. $\mathrm{Na}^{+}$transport in plants. FEBS Letters, v.581, p.2247-2254, 2007.
ARNON, D.I. Copper enzymes in isolated chloroplasts. Polyphenoloxidases in Beta vulgaris. Plant Physiology, v.24, p.1-15, 1949.

ASHRAF, M.; AHMAD, S. Influence of sodium chloride on ion accumulation, yield components and fibre characteristics in salttolerant and salt-sensitive lines of cotton (Gossypium hirsutum L.). Field Crops Research, v.66, p.115-127, 2000.

ASHRAF, M.; HARRIS, P.J.C. Potential biochemical indicators of salinity tolerance in plants. Plant Science, v.166, p.3-16, 2004.

BARROS, L. de M.; CAVALCANTI, J.J.V.; PAIVA, J.R. de; CRISÓSTOMO, J.R.; CORRÊA, M.P.F.; LIMA, A.C. Seleção de clones de cajueiro-anão para o plantio comercial no estado do Ceará. Pesquisa Agropecuária Brasileira, v.35, p.2197-2204, 2000.

BATES, L.S.; WALDREN, R.P.; TEARE, I.D. Rapid determination of free proline for water stress studies. Plant and Soil, v.39, p.205-207, 1973.

BEZERRA, I.L.; GHEYI, H.R.; FERNANDES, P.D.; SANTOS, F.J. de S.; GURGEL, M.T.; NOBRE, R.G. Germinação, formação de porta-enxertos e enxertia de cajueiro anão precoce, sob estresse salino. Revista Brasileira de Engenharia Agrícola e Ambiental, v.6, p.420-424, 2002.

BEZERRA, M.A.; LACERDA, C.F. de; GOMES FILHO, E.; ABREU, C.E.B. de; PRISCO, J.T. Physiology of cashew plants grown under adverse conditions. Brazilian Journal of Plant Physiology, v.19, p.449-461, 2007.

BRADFORD, M.M. A rapid and sensitive method for the quantitation of microgram quantities of protein utilizing the principle of protein-dye binding. Analytical Biochemistry, v.72, p.248-254, 1976.

BRAY, E.A.; BAILEY-SERRES, J.; WERETILNIK, E. Responses to abiotic stresses. In: BUCHANAN, B.B.; GRUISSEM, W.; JONES, R.L. (Ed.). Biochemistry and molecular biology of plants. Rockville: American Society of Plant Physiologists, 2000. p.1158-1249.

CARNEIRO, P.T.; CAVALCANTI, M.L.F.; BRITO, M.E.B.; GOMES, A.H.S.; FERNANDES, P.D.; GHEYI, H.R. Sensibilidade do cajueiro anão precoce ao estresse salino na pré-floração. Revista Brasileira de Ciências Agrárias, v.2, p.150-155, 2007.

DASGAN, H.Y.; AKTAS, H.; ABAK, K.; CAKMAK, I. Determination of screening techniques to salinity tolerance in tomatoes and investigation of genotype responses. Plant Science, v.163, p.695-703, 2002.

ESTAÑ, M.T.; MARTINEZ-RODRIGUEZ, M.M.; PEREZALFOCEA, F.; FLOWERS, T.J.; BOLARIN, C. Grafting raises the salt tolerance of tomato through limiting the transport of sodium and chloride to the shoot. Journal of Experimental Botany, v.56, p.703-712, 2005.

FERREIRA-SILVA, S.L.; SILVEIRA, J.A.G; VOIGT, E.L.; SOARES, L.S.P; VIÉGAS, R.A. Changes in physiological indicators associated with salt tolerance in two contrasting cashew rootstocks. Brazilian Journal of Plant Physiology, v.20, p.51-59, 2008.

FLOWERS, T.J. Improving crop salt tolerance. Journal of Experimental Botany, v.55, p.307-319, 2004. 
GARCÍA-SÁNCHEZ， F.; JIFON， J.L.; CARVAJAL， M.; SYVERTSEN, J.P. Gas exchange, chlorophyll and nutrient contents in relation to $\mathrm{Na}^{+}$and $\mathrm{Cl}^{-}$accumulation in 'Sunburst' mandarin grafted on different Rootstocks. Plant Science, v.162, p.705-712, 2002.

GRATTAN, S.R.; GRIEVE, C.M. Salinity mineral nutrient relations in horticultural crops. Scientia Horticulturae, v.78, p.127-157, 1999.

HOAGLAND, D.R.; ARNON, D.I. The water-culture method for growing plants without soil. Berkeley: California Agricultural Experiment Station, 1950. 32p. (Circular, 347).

LUTTS, S.; KINET, J.M.; BOUHARMONT, J. Effects of salt stress on growth, mineral nutrition and proline accumulation in relation to osmotic adjustment in rice (Oryza sativa L.) cultivars differing in salinity tolerance. Plant Growth Regulation, v.19, p.207-218, 1996.

MAATHUIS, F.J.M.; AMTMANN, A. $\mathrm{K}^{+}$nutrition and $\mathrm{Na}^{+}$toxicity: the basis of cellular $\mathrm{K}^{+} / \mathrm{Na}^{+}$ratios. Annals of Botany, v.84, p.123-133, 1999.

MUNNS, R.; TESTER, M. Mechanisms of salinity tolerance. Annual Review of Plant Biology, v.59, p.651-681, 2008.

PAIVA, J.R. de; CARDOSO, J.E.; BARROS, L. de M.; CRISÓTOMO, J.R.; CAVALCANTI, J.J.V.; ALENCAR, E. da S. Clone de cajueiro precoce BRS 226 ou PLANALTO: nova alternativa para o plantio na Região semi-árida do Nordeste. Fortaleza: Embrapa Agroindústria Tropical, 2002. (Embrapa Agroindústria Tropical. Comunicado Técnico, 78).

PAIVA, J.R. de; CARDOSO, J.E.; MESQUITA, A.L.M.; CAVALCANTI, J.J.V.; SANTOS, A.A. dos. Desempenho de clones de cajueiro-anão precoce no semi-árido do Estado do Piauí. Revista Ciência Agronômica, v.39, p.295-300, 2008.

PARANYCHIANAKIS, N.V.; ANGELAKIS, A.N. The effect of water stress and rootstock on the development of leaf injuries in grapevines irrigated with saline effluent. Agricultural Water Management, v.95, p.375-382, 2008.

PEOPLES, M.B.; FAIZAH, A.W.; RERKASEM, B.; HERRIDGE, D.F. Methods for evaluating nitrogen fixation by nodulated legumes in the field. Canberra: Australian Centre for International Agricultural Research, 1989. 76p. (Aciar. Monography, 11).

SILVEIRA， J.A.G.; VIÉGAS， R.D.; ROCHA， I.M.A. da; MOREIRA, A.C.D.M.; MOREIRA, R.D.; OLIVEIRA, J.T.A. Proline accumulation and glutamine synthetase activity are increased by salt-induced proteolysis in cashew leaves. Journal of Plant Physiology, v.160, p.115-123, 2003.

VIÉGAS, R.A.; SILVEIRA, J.A.G. da; LIMA JÚNIOR, A.R. de; QUEIROZ, J.E.; FAUSTO, M.J.M. Effects of NaCl-salinity on growth and inorganic solute accumulation in young cashew plants. Revista Brasileira de Engenharia Agrícola e Ambiental, v.5, p.216-222, 2001.

VIÉGAS, R.A.; SILVEIRA, J.A.G. da; SILVA, L.M. de M.; VIÉGAS, P.R.A.; QUEIROZ, J.E.; ROCHA, I.M.A. Redução assimilatória de $\mathrm{NO}_{3}^{-}$em plantas de cajueiros cultivados em meio salinizado. Revista Brasileira de Engenharia Agrícola e Ambiental, v.8, p.189-195, 2004.

VOIGT, E.L.; ALMEIDA, T.D.; CHAGAS, R.M.; PONTE, L.F.A.; VIÉGAS, R.A.; SILVEIRA, J.A.G. Source-sink regulation of cotyledonary reserve mobilization during cashew (Anacardium occidentale) seedling establishment under $\mathrm{NaCl}$ salinity. Journal of Plant Physiology, v.166, p.80-89, 2009.

WAHOME, P.K.; JESCH, H.H.; GRITTNER, I. Mechanisms of salt stress tolerance in two rose rootstocks: Rosa chinensis Major and R. rubiginosa. Scientia Horticulturae, v.87, p.207-216, 2001.

WHITE, P.J.; BROADLEY, M.R. Chloride in soils and its uptake and movement within the plant: a review. Annals of Botany, v.88, p.967-988, 2001.

Recebido em 12 de novembro de 2008 e aprovado em 4 de março de 2009 